\title{
Evolution of shear zones in granular packings under pressure
}

\author{
Mahnoush Madani, ${ }^{1}$ Maniya Maleki, ${ }^{1}, 2$ János Török, ${ }^{3}$ and M. Reza Shaebani, ${ }^{4}$, \\ ${ }^{1}$ Department of Physics, Institute for Advanced Studies in Basic Sciences, Zanjan 45137-66731, Iran \\ ${ }^{2}$ Optics Research Center, Institute for Advanced Studies in Basic Sciences, Zanjan 45137-66731, Iran \\ ${ }^{3}$ MTA-BME Morphodynamics Research Group, Department of Theoretical Physics, \\ Budapest University of Technology and Economics, Budapest H-1111, Hungary \\ ${ }^{4}$ Department of Theoretical Physics \& Center for Biophysics, \\ Saarland University, 66041 Saarbrücken, Germany
}

\begin{abstract}
Stress transmission in realistic granular media often occurs under external load and in the presence of boundary slip. We investigate shear localization in a split-bottom Couette cell with smooth walls subject to a confining pressure experimentally and by means of numerical simulations. We demonstrate how the characteristics of the shear zone, such as its center position and width, evolve as the confining pressure and wall slip modify the local effective friction coefficient of the material. For increasing applied pressure, the shear zone evolves toward the center of the cylinder and grows wider and the angular velocity reduces compared to the driving rate of the bottom disk. Moreover, the presence of slip promotes the transition from open shear zones at the top surface to closed shear zones inside the bulk. We also systematically vary the ratio of the effective friction near the bottom plate and in the bulk in simulations and observe the resulting impact on the surface flow profile. Besides the boundary conditions and external load, material properties such as grain size are also known to influence the effective friction coefficient. However, our numerical results reveal that the center position and width of the shear zone are insignificantly affected by the choice of the grain size as far as it remains small compared to the radius of the rotating bottom disk.
\end{abstract}

\section{INTRODUCTION}

Driven granular systems exhibit intriguing phenomena such as segregation, pattern formation, granular capillarity, and shear thickening and localization [1 8$]$. The latter is of great scientific, industrial, and geophysical importance. In slowly sheared granular materials, where lasting contacts play the main role in momentum exchange, one often observes a spatially inhomogeneous flow with the strain being localized in narrow shear zones [6, $7,9-13$. The flow profile and shear stress are basically independent of the shear rate in this regime so that the shear localization cannot be fully captured by a constitutive stress-strain relation. In contrast to this regime, momentum transfer in fast granular flows is dominated by both lasting contacts and collisions. Here, the local flow rate can be characterized by a dimensionless inertial number which relates the shear stress to the shear rate, enabling to construct a stress-strain constitutive law and successfully describe the flow profile 14, 15.

When slowly shearing the boundaries of a granular system, narrow shear zones with a typical width of a few particle diameters form near the moving boundaries. The experiments on granular flows in Couette cells revealed that the shape of the wall-localized flow profile is independent of both filling height and shear rate and is solely determined by the characteristics of the granular microstructure [9].

Nevertheless, shear zones away from the boundaries were also generated later in shear experiments in splitbottom Couette geometries, where the rough bottom plate of the cylindrical cell was split in two parts that rotate relative to each other $[11,13,16]$. In such geome- tries, the shear band is pinned in the bottom to the split position $r=R_{c}$ far from the side walls at $r=R$ (see Fig.11). The shear zone is relatively wide as it takes advantage of the gravity to reach the top free surface through the bulk of the granular pile. In contrast to wall-localized shear zones, here the shape of the flow profile is governed by the filling height $H$. Additionally, the axial symmetry of the cylindrical split-bottom cell implies that the split position is another influential parameter in determining the position and width of the shear zone. For small driving rates $\Omega$ of the rotating bottom disk, the normalized flow profile is independent of $\Omega$. Starting from the split, the shear zone gradually grows wider and evolves toward the center of the cylinder with increasing height. Whether it reaches the top free surface depends on the ratio $H / R_{c}$; in deep granular layers the shear zone forms a dome-like shape in the bulk while in shallow layers it reaches the top free surface 13, 16 18.

In shallow-layer experiments, it was shown that the axial angular velocity at the free surface $\omega_{0}-$ defined as $\omega_{0} \equiv \lim _{r \rightarrow 0} \omega(r ; h=H)$ - equals the driving rate $\Omega$ of the rough bottom disk [11, 12]. Moreover, the surface velocity profile $\omega(r)$ as a function of the radial coordinate $r$ is well fitted by an error function $\frac{\omega(r)}{\Omega}=\frac{1}{2}\left(1-\operatorname{erf}\left\{\frac{r-R_{s}}{\delta}\right\}\right)$, with $R_{s}$ and $\delta$ being the center of the shear zone and its width, respectively. The center position follows $\frac{R_{s}}{R_{c}}=1-\left(\frac{H}{R_{c}}\right)^{5 / 2}$ and the width grows as a power-law $\delta \sim H^{2 / 3}$, i.e. faster than diffusion but slower than linear. A continuum constitutive model for dense granular flows was shown to capture the main features of the flow profile in shallow-layer experiments 


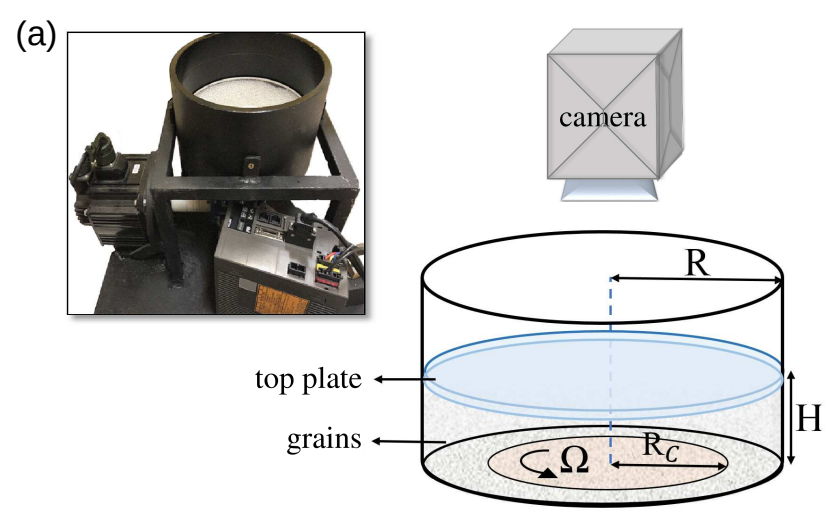

(b)

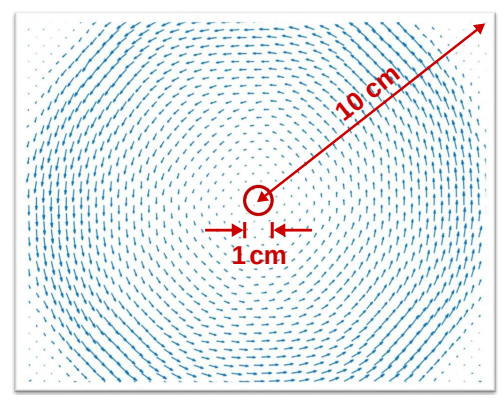

FIG. 1. (a) Sketch of the split-bottom Couette cell. (b) Displacement field of the grains from PIV analysis obtained from the subtraction of two successive configurations taken from the top surface of the granular pile. The red circle marks the region over which the axial angular velocity $\omega_{0}$ is measured.

in the split-bottom Couette geometry [19, 20]. However, axial slip occurs with increasing the filling height $H$ [13, 16]; because of shearing between horizontal granular layers, $\omega_{0}$ decreases and the velocity profile at the surface deviates from the error function shape. Above $H / R_{c} \sim 0.6-0.7, \omega_{0} \rightarrow 0$ and the surface profile vanishes.

So far, the Couette flow experiments to study shear localization in granular media have been carried out in cells with an open top boundary. Much less is known about the evolution of shear zones in more realistic media (as, for example, in geological processes) where the system is confined or subjected to an external pressure. Under such conditions, the dilation behavior and microstructure of granular materials change 21 27] which is expected to influence the flow profile. It was predicted based on an energy-dissipation variational approach that applying an external pressure should result in a similar behavior as observed with increasing filling height [17].

Another point is that the role of effective friction coefficient near the bottom plate has yet to be resolved. In general, the effective friction in the bulk of the granular pile $\mu_{\text {bulk }}^{\text {eff }}$ differs from the effective friction near the rotating disk $\mu_{\mathrm{bottom}}^{\mathrm{eff}}$. One expects that the flow profile changes upon varying the ratio $\mu_{\mathrm{bottom}}^{\mathrm{eff}} / \mu_{\mathrm{bulk}}^{\mathrm{eff}}$, since friction plays a key role in stress transmission in granular media 28 32] and particularly inside wide shear bands [33] (For a given $\mu_{\text {bottom }}^{\text {eff }} / \mu_{\text {bulk }}^{\text {eff }}$ ratio, the actual value of $\mu_{\text {bulk }}^{\text {eff }}$ or $\mu_{\text {tf }}^{\text {eff }}$ does not influence the shear zone properties [10, 17]). It is however hard to tune the ratio in practice; even gluing a layer of grains to the walls with the same grains as in the bulk does not guarantee that the effective frictions are equal [10]. A systematic study of the parameters such as size and shape of the particles is necessary to better understand the role of material properties on the effective friction coefficient and shear deformation of granular materials. It has been shown that shearing of non-spherical grains in split-bottom ge- ometries induces particle alignment (which reduces the effective friction) and leads to heap formation near the rotation axis 34 36]; however, the role of particle size is yet to be explored.

Furthermore, by considering rough boundaries in the previous experiments in Couette geometries, the possibility of wall slip has been often avoided. Nevertheless, wall slip occurs in real granular flows and it is not clear whether, and how, the presence of slip near the walls affects the flow profile.

In this paper, we investigate the formation of shear band in a split-bottom Couette cell under external pressure $P$ and in the presence of slip between the grains and the bottom plate. By varying the weight of a top plate, we change the relative difference between the stress components of successive layers of grains, which affects the center position and width of the shear zone. The smooth bottom plate in our experimental setup allows for axial slip at the bottom layer, which significantly influences the strength of the surface flow profile and expedites the transition to closed shear zones inside the bulk. We also numerically study the role of the wall-to-bulk ratio of the effective friction coefficient as well as the particle size on shear localization. Therefore, by investigating the individual roles of the factors mentioned above, our main plan is to better understand the role of boundary constraints and conditions - such as wall slip and roughness and applied loads - on shear localization in granular flows.

\section{EXPERIMENTAL SETUP}

The experimental setup is similar to the split-bottom Couette cell previously used in experiments on granular flows 11, 12] (see Fig. 11). It consists of a rotating bottom disk of radius $R_{c}=75 \mathrm{~mm}$ and a stationary bottom ring that is attached to a cylinder of radius $R=100 \mathrm{~mm}$. 

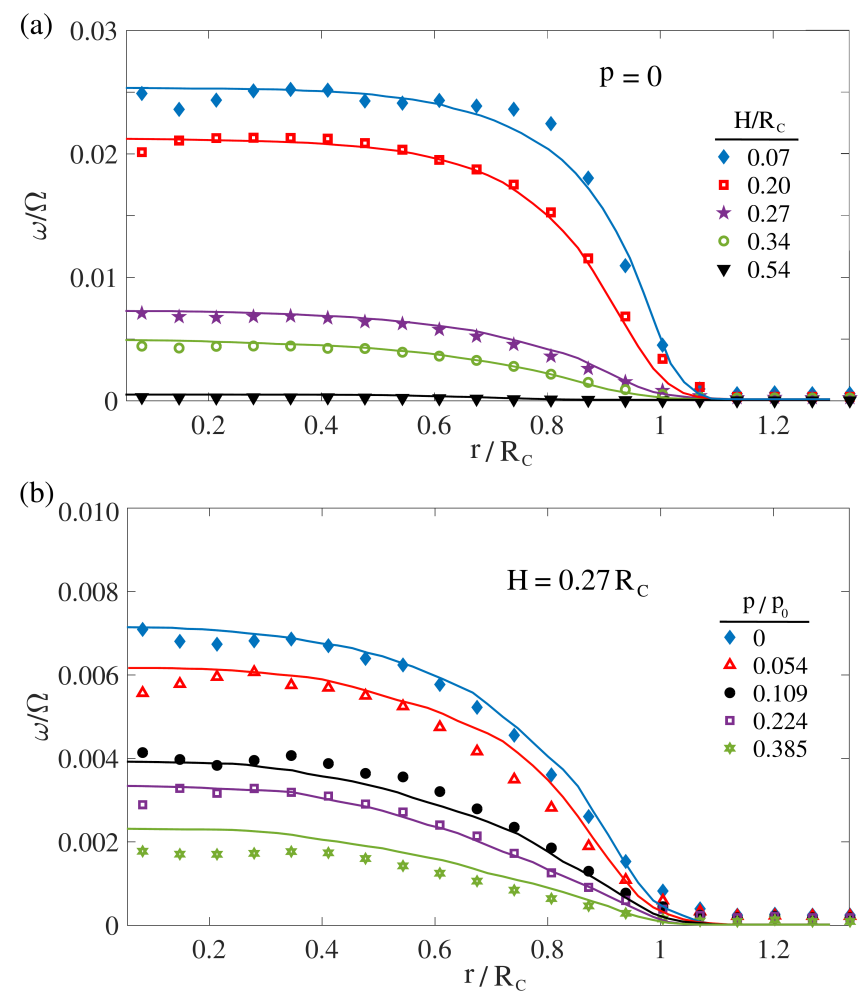

FIG. 2. Angular surface velocity $\omega$, normalized by the driving rate $\Omega$ of the bottom disk, versus the radial coordinate $r$, for (a) $P=0$ and various filling heights, and (b) $H=0.27 R_{c}$ and different values of the external pressure. $P_{0}$ is the reference pressure applied by the pile of grains with height $H$ for comparison. Symbols represent the experimental results and lines are obtained from the variational approach via Eq. (2).

To apply an external pressure on the packing, we put a plexiglass plate with a mass ranging from 50 to $550 \mathrm{gr}$ on top of the granular layer. For ease of comparison, we choose the pressure applied by a pile of grains with filling height $H$ as the reference pressure $P_{0}$. Note that the applied pressure is in the order of the mean hydrostatic pressure of the granular pile and the anisotropic contact network (i.e. the network constructed by the normal vectors to contact planes) influenced by gravity is still far from being an ideal isotropic packing [37].

The cell is filled with grains up to height $H$. The average diameter of the glass beads is $2 \mathrm{~mm}$ with size polydispersity of about $15 \%$. This prevents crystallization effects near walls (i.e. shear-induced layering of equalsized particles) that can affect the flow profile [9, 38]. The bottom plate and container walls are smooth, allowing for axial slip of the bottom layer of grains. The size of the gap at the split is less than $400 \mu \mathrm{m}$, which is much smaller than the minimal particle size, thus, no particle can escape.

The bottom disk is rotated at angular velocity $\Omega$. The driving rate should be small enough to avoid ratedependent stresses [39]. The results reported here belong (a)

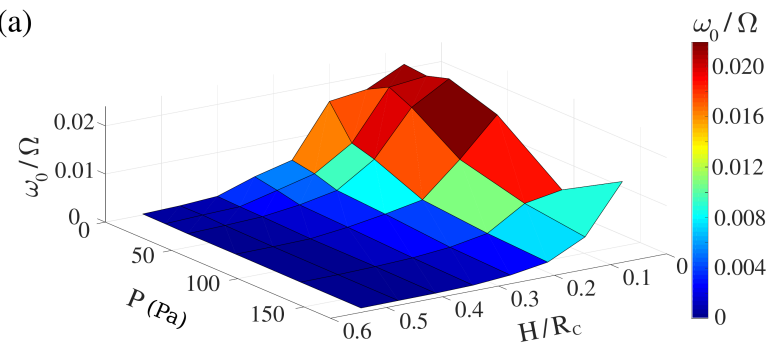

(b)

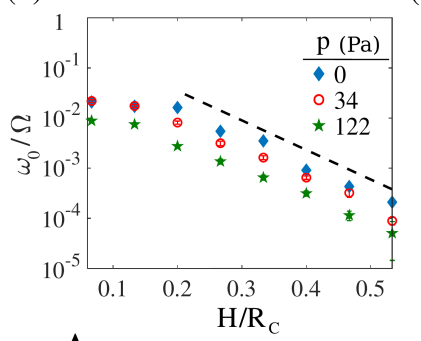

(c)
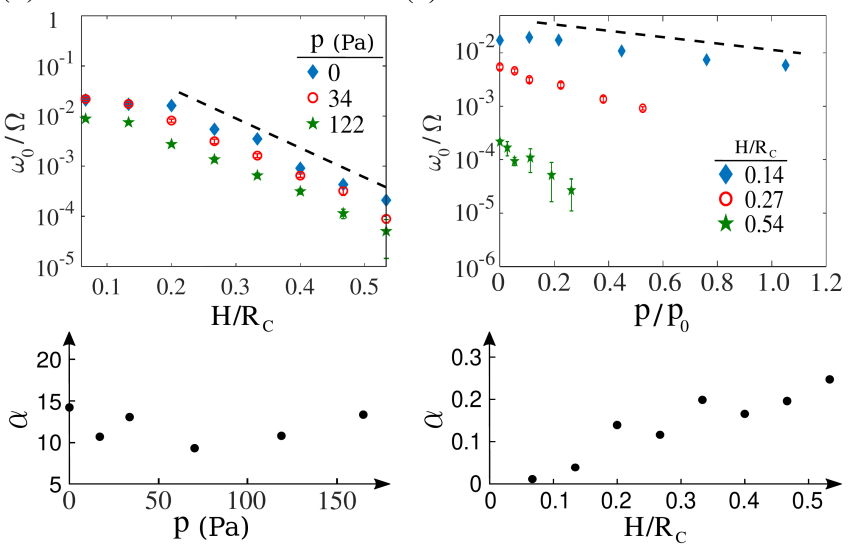

FIG. 3. (a) Axial angular velocity $\omega_{0}$ in the $(H, P)$ space. (b) top: $\omega_{0}$ vs $H$ at different applied pressures. The dashed line shows an exponential decay as a guide to eye. bottom: Decay exponent $\alpha$ obtained from fitting an exponential function to the tail of $\omega_{0}-H$ curves at different $P$ values. (c) top: $\omega_{0}$ vs $P$ at different filling heights. bottom: Exponent $\alpha$ of the exponential decay of $\omega_{0}-P$ tail at different filling heights $H$. The surface plot in panel (a) and symbols in top panels of (b) and (c) represent the experimental data.

to $\Omega=0.13 \mathrm{rad} / \mathrm{s}$. After the flow reaches a steady state in a few seconds, we measure the flow profile at the top surface. The surface flow is monitored from above by a Casio camera with a CMOS sensor and frame rate $30 \mathrm{~s}^{-1}$. The experiments are carried with and without the top plate. Because of using a camera with high pixel resolution of $\sim 2.5 \mu \mathrm{m}$, the observation time of the surface flow that is required to reconstruct the particle displacement field is relatively short (ranging from a few seconds to a couple of minutes depending on the choice of $P$ and $H$ parameters). To obtain the mean angular velocity $\omega(r)$ at the top surface as a function of the radial coordinate $r$, particle image velocimetry method (PIV) is used, where the average angular cross-correlation function is determined in terms of $r$ for temporally separated frames. In order to obtain the axial angular velocity $\omega_{0}$ at the top surface, we average the angular velocities within a small circle of radius $5 \mathrm{~mm}$ around the cylinder axis; see the red marked circle in Fig.1(b). 

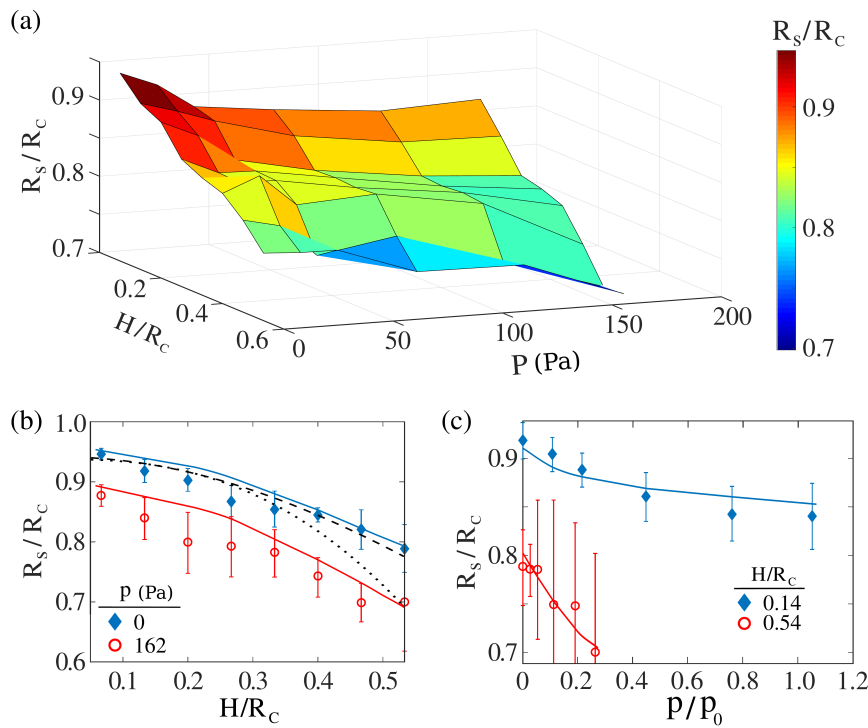

(d)

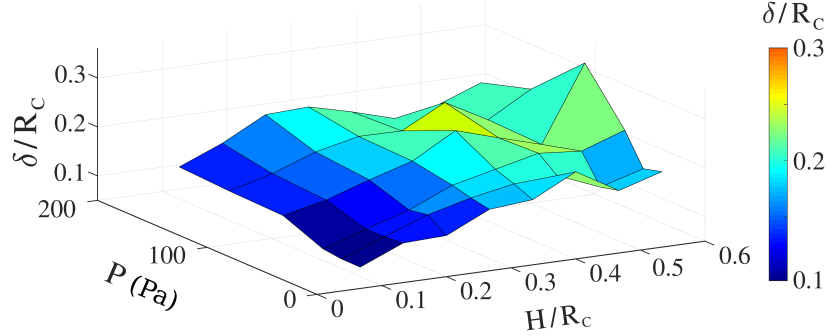

(e)
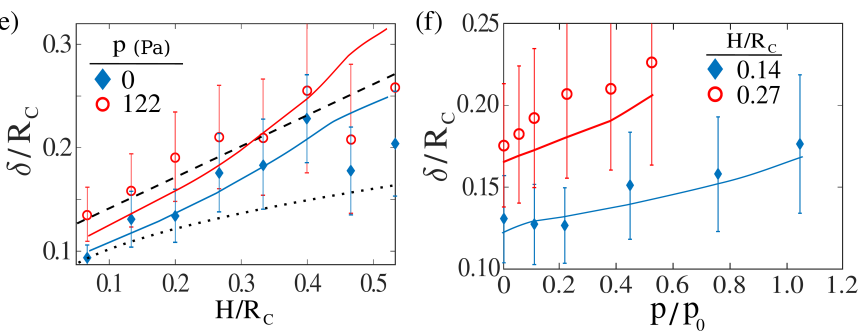

FIG. 4. (a) Shear zone position $R_{s}$ at the top surface in terms of the filling height $H$ and applied pressure $P$. (b) $R_{s}$ vs $H$ at different values of $P$. The dotted line represents $R_{s} / R_{c} \propto 1-\left(\frac{H}{R_{c}}\right)^{5 / 2}$ and the dashed line is a Gaussian fit to the free-surface (i.e. $P=0$ ) data. (c) $R_{s}$ vs $P$ at different values of $H$. (d) Shear zone width $\delta$ at the top surface in terms of $H$ and $P$. (e), (f) $\delta$ vs $H$ or $P$ for different choices of the other control parameter. The surface plots in panels (a) and (b) and symbols in all lower panels represent experimental results while solid lines are obtained numerically. The dashed and dotted lines in panel (e) correspond to $\delta / R_{c} \sim H / R_{c}$ and $\delta / R_{c} \sim \sqrt{H / R_{c}}$, respectively.

\section{RESULTS}

First we investigate the role of wall slip on the velocity profiles. According to the previous experimental reports in split-bottom Couette cells with a rough bottom plate, no axial slip occurs in shallow layers $\left(H / R_{c} \leq 0.45\right)$ [12, 13, 16]; the inner region above the central disk rotates as a solid while the outer part is stationary, and the angular velocity profile at the free surface is well fitted by the error function. At intermediate filling heights $0.45<H / R_{c} \leq 0.65-0.7$, deviations from the ideal error function shape start to appear and the axial angular velocity $\omega_{0}$ at the top surface decreases. The axial slip results from shear between horizontal layers inside the bulk, while the bottom layer still rotates at the same rate as the bottom plate because of the rough surface of the bottom disk 13. Eventually, the flow pattern at the free surface disappears for deep layers $H / R_{c}>0.7, \omega_{0}$ approaches zero, and the shear zone is fully buried inside the bulk. We focus on the shallow-layer regime in our experiments with a smooth bottom plate. In the absence of the top plate, the flow profile at the free surface is shown in Fig.2(a) for various filling heights. It can be seen that even at extremely shallow layers, the axial angular velocity $\omega_{0}$ is nearly two orders of magnitude smaller than $\Omega$. This indicates that the majority of the slip between the stationary and the moving parts occurs at the bottom plate. It was shown in [10] that the smooth walls can be attractors of shear zones. $\omega_{0}$ further decreases with increasing $H$ so that the surface flow practically disappears for $H / R_{c} \geq 0.45$. The shape of the surface flow profile also starts deviating from the error function at very shallow layers $H / R_{c} \simeq 0.1$.

The axial slip at the bottom smooth plate reduces the shear strength of the bulk material and weakens the flow profile. For the material properties used in our setup, we observe a considerable level of slip with $\omega_{0} / \Omega$ being around 0.03 for extremely shallow layers. Still, the bulk is sheared strong enough so that the shear zone can proceed to the higher layers of grains. Then, an additional axial slip due to shear between horizontal layers inside the bulk further decreases $\omega_{0}$ and the strength of the surface profile. As a result, the transition from shear zones reaching the free surface to closed shear zones inside the bulk occurs at smaller values of $H$ compared to the case of rough bottom walls.

Next, we examine the surface flow profiles in the presence of the top glass plate. A few exemplary velocity profiles at $H / R_{c}=0.27$ and different external pressures $P$ are shown in Fig.2(b). Increasing the applied pressure acts similarly to rising the filling height: it reduces $\omega_{0}$ and weakens the strength of the surface flow. Fig.3(a) shows the phase diagram of $\omega_{0}$ in the $(H, P)$ space. Approaching the plateau region at high $H$ or $P$ values, the shear zone is completely buried inside the bulk of the granular pile. A closer look at the behavior of $\omega_{0}$ in Figs.3(b),(c) reveals that the tail of $\omega_{0}$ versus $H$ or $P$ decays nearly exponentially [13] with a constant $\alpha$ that shows no systematic dependence on the pressure $P$ but gradually increases with $H$. Indeed, the relative difference between 
the components of the shear stress $\sigma_{\mathrm{ij}}$ of two successive horizontal layers of grains depends on their height difference and is independent of the applied isotropic pressure or the absolute height of the pile above the two layers. That is why the decay of axial slip due to shear between horizontal layers is not affected by the choice of $H$ or $P$. Note that the increase of $\alpha$ with $H$ in Fig. 3( 3 (c) is caused by the increase of the reference pressure $P_{0}$ with $H$.

Because of the strong slip at the bottom disk, shearing even a very shallow layer leads to $\omega_{0}<\Omega$ and a modified flow profile at the top surface. Figure2 shows that with increasing $P$ or $H$ the profiles become more asymmetric with respect to the center position of the shear zone, but it is yet clear that the profile grows wider and the center position shifts toward the cylinder axis. An errorlike function with four fit parameters was previously used to capture the asymmetric form of the flow profiles [16]. However, we checked that neither this form nor a logistic function provide a satisfactory fit to our data. Alternatively, we more precisely determine the characteristics of the shear zone from the shear rate information. For example, the center of shear zone can be characterized as the radial distance $R_{s}$ at which the shear rate has its maximum, i.e. $\mathrm{d} \epsilon_{r \theta} /\left.\mathrm{d} r\right|_{r=R_{s}}=0$ with $\epsilon$ being the rate of the strain tensor. One can also quantify the width $\delta$ of the shear zone by, e.g., calculating the variance of the shear rate $\mathrm{d} \omega / \mathrm{d} r$ around the peak at $r=R_{s}$. Both $R_{s}$ and $\delta$ parameters can be alternatively deduced from fitting the surface flow profile to an error function, which leads to qualitatively similar results and trends in terms of $P$ or $H$. Figures 4 (a),(d) summarize the behavior of $R_{s}$ and $\delta$ in the $(H, P)$ space. The relative decrease of $R_{s}$ versus $H$ is slower than the power-law $\frac{R_{c}-R_{s}}{R_{c}} \propto\left(\frac{H}{R_{c}}\right)^{5 / 2}$ observed for shallow-layer shear in split-bottom Couette cells with a rough bottom disk [12. In Fig. 4(b) we show a fit to the free surface $(P=0)$ data as an example. Dramatic deviations can be observed for deeper layers. We find that a Gaussian decay $R_{s} / R_{c} \propto \exp \left[-\frac{\left(H / R_{c}\right)^{2}}{2 a^{2}}\right]$ (with $a$ being a fit parameters) better captures the behavior [see the dashed line in Fig.4(b)]. This holds also for other values of the external pressure as well as for $R_{s}$ vs $P$ curves at different filling heights. For the broadening of the shear zone with $H$, we find that $\delta$ grows faster than $\sqrt{H / R_{c}}$ but slower than $H / R_{c}$ for different values of the external pressure. The corresponding lines for the case of $P=0$ are shown in Fig.4(e). Furthermore, when fitting $\delta / R_{c}$ vs $P$ to a power-law, the decay exponent again lies within $[0.5,1]$. It was similarly shown in rough-bottom geometries that the broadening of the shear zone at the free surface of the granular pile is faster than diffusion but remains slower than a linear growth [11].

\section{NUMERICAL APPROACH}

In order to provide physical insight into how isotropic pressure influences the flow profile shape, we perform numerical simulations based on a variational minimization approach 40 43] that has been successfully applied to describe strain localization in granular materials 10, 17, 18, 44 46]. Despite the availability of efficient numerical tools for large-scale discrete element method simulations [47, 48], the variational approach is a computationally cheap way of obtaining the flow profile in symmetric geometries with simple boundary conditions. The main idea behind the variational approach is that the shear band forms along a path that minimizes the dissipation rate and complies with the boundary conditions. The cylindrical symmetry of the setup enables us to reduce the problem to a two-dimensional one. We consider a discrete 2D square lattice with a lattice size equal to the grain diameter. Denoting the radial coordinate of the shear band at height $h$ inside the bulk with $r(h)$, the boundary condition is $r(0)=R_{c}$. According to the original narrow-shear-band variant of the model [17], the width of the shear band can be only one unit cell. Thus, two solid blocks slide past each other and the dissipation rate $\mathrm{d} \gamma$ for a ring of sliding area with radius $r(h)$ is given by

$$
\mathrm{d} \gamma=\sigma_{\mathrm{tn}} v(r) \mathrm{d} s,
$$

where $\sigma_{\text {tn }}$ is the shear stress, $v(r)=\Omega r(h)$ the sliding velocity between the blocks, and $\mathrm{d} s=2 \pi r(h) \mathrm{d} l$ the sliding area $\left(\mathrm{d} l=\sqrt{(\mathrm{d} h)^{2}+(\mathrm{d} r)^{2}}\right)$. To obtain the total dissipation rate, $\mathrm{d} \gamma$ needs to be integrated over the whole surface of the shear band. Thus, up to a constant prefactor, the integral to be minimized can be expressed as

$$
\int_{\text {path }}^{r^{2} \sigma_{\text {tn }} \mathrm{d} l=\min ,}
$$

which results in an instantaneous narrow shear band. However, the material properties in the vicinity of the shear band is expected to change in practice. In a generalized fluctuating-band version of the model [18], local fluctuations of the path due to random structural changes in the vicinity of the shear band have been introduced. As a result, the minimal path would slightly change in the next instance and the final shear profile is obtained by an ensemble average over all instantaneous narrow shear bands. Such a self-organized process allows for a finite width of the shear zone.

We assume that the effective friction coefficient in the vicinity of the bottom plate $\mu_{\mathrm{bottom}}^{\mathrm{eff}}$ differs from that of the bulk $\mu_{\text {bulk }}^{\text {eff }}$ in general. The ratio $\mu_{\text {rel }}=\mu_{\text {bottom }}^{\text {eff }} / \mu_{\text {bulk }}^{\text {eff }}$ is varied within $[0,10]$ in our simulations to investigate the role of axial slip at the bottom smooth plate. The initial and updated random strengths of the lattice sites follow the probability distribution $P\left(\mu^{\mathrm{eff}}\right)$ reported in $\operatorname{Ref}[49]$. 


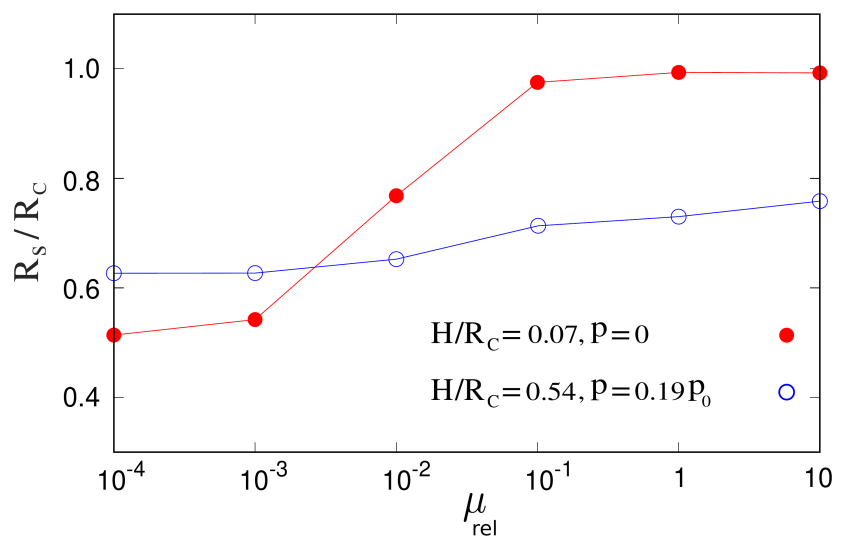

FIG. 5. Shear zone position $R_{s}$ at the top surface, scaled by the split position $R_{c}$, as a function of the relative friction coefficient $\mu_{\text {eff }}$ for different values of $P$ and $H$. The results are obtained from the variational approach via Eq (2).

Because of the continuous agitation of the system, the Janssen effect plays no role here and we can assume a pressure $\rho g(H-h)+P$ at height $h$ in the bulk. Then the shear stress is obtained as $\sigma_{\text {tn }}=\mu^{\text {eff }}(\rho g(H-h)+P)$. Therefore, the isotropic pressure and the filling height influence the shear stress (and, thus, the variation integral) in a similar way. This explains why the same trends in terms of $P$ or $H$ are observed for the characteristics of the shear zone in our experiments (They are however not interchangeable because $H$ additionally sets the variational integration limit). To calculate the optimal path, most of the model parameters are fixed by the geometry and boundary conditions. The only remaining free parameters are the lattice size $d$ and the ratio of the effective friction coefficients of the bottom disk and bulk, $\mu_{\text {rel }}$. We first choose $d=2 \mathrm{~mm}$ and tune $\mu_{\text {rel }}$ as a free parameter to reproduce the experimental results and then study the role of grain size and wall slip by varying $d$ and $\mu_{\text {rel }}$ parameters.

Figure2(a) shows that the flow profiles obtained by tuning the free parameter $\mu_{\text {rel }}$ for each set of $(H, P)$ parameters satisfactorily capture the trends observed in experiments. The resulting friction ratio $\mu_{\text {rel }}$ slightly varies (within $[0.05,0.1]$ ), since variation of $H$ or $P$ could influence the arrangement and packing fraction of particles near the bottom plate and change the interparticle contact network (i.e. the positions of the contacts and their orientations). This leads to the modification of the effective friction coefficient $\mu_{\mathrm{bottom}}^{\mathrm{eff}}$. The influence of $H$ or $P$ on the fitting parameter $\mu_{\text {rel }}$ is more pronounced at shallow layers or low confining pressures. At higher values of $H$ or $P$, even fitting all flow profiles with a single optimal choice of $\mu_{\text {rel }}$ leads to insignificant deviations from experimental trends. For example, all the flow profiles in Fig.2(b) for a moderate filling height $H / R_{c}=0.27$ are obtained using $\mu_{\mathrm{rel}}=0.1$; the agreement is still satisfac-

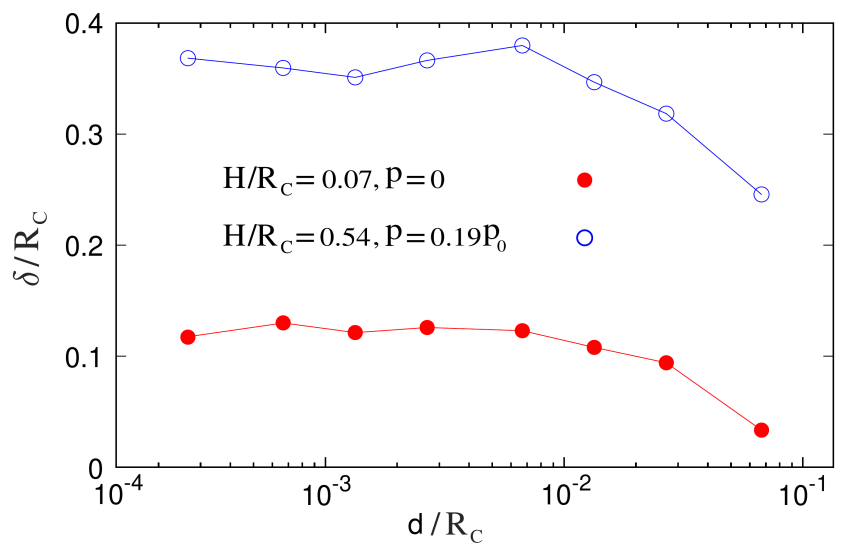

FIG. 6. Width of the shear zone at the top surface, scaled by the split position, in terms of the scaled grain size $d / R_{c}$ for different values of filling height and external pressure. The results are obtained from the variational approach via Eq (2).

tory. We also checked that the error-function shape is recovered for $\mu_{\text {rel }}=1$ in shallow layers, independent of the actual value of $\mu_{\mathrm{bulk}}^{\mathrm{eff}}=\mu_{\mathrm{bottom}}^{\mathrm{eff}}$. The predicted center position and width of the shear zone shown in Fig. 4 match well the experimental trends.

Next we systematically examine the role of effective friction at the bottom layer on shear localization, by varying $\mu_{\text {rel }}=\mu_{\text {bottom }}^{\mathrm{eff}} / \mu_{\mathrm{bulk}}^{\mathrm{eff}}$ by several orders of magnitude. Overall, decreasing $\mu_{\text {rel }}$ induces a stronger axial slip at the bottom disk and shifts the center position of the shear zone towards the cylinder axis. In the extreme regime of very shallow layers at low confining pressures, the inner region above a rough disk rotates as a solid and the center of the shear zone is almost above the split, i.e. $R_{s} \simeq R_{c}$. An evident transition in the center position towards the cylinder axis can be observed in this case upon decreasing $\mu_{\text {rel }}$ (see Fig. (5). The change is much less pronounced for deep layers and high pressures, where the contribution of axial slip due to shear between horizontal layers of grains is significant. We observe no systematic dependence of the shear zone width on $\mu_{\text {rel }}$.

Finally, we vary the lattice size $d$ in the variational calculations to see how grain size affects the position and width of the shear zone. By changing $d$ by more than two orders of magnitude while keeping the split position fixed at $R_{c}=75 \mathrm{~mm}$, we observe no dependence of the center position on the relative grain size $d / R_{c}$. Also the shear zone width is not affected in small grain size regime. However, the width decreases by increasing the coarse-graining size above $1 \%$ of the container radius, as shown in Fig.66. The energy dissipation cost of changing the narrow shear band path increases with the lattice size and eventually surpasses the gain by following the local changes in the material strength. This reduces the fluctuation range of the ensemble of instantaneous narrow shear bands for relatively large grains. 


\section{CONCLUSION}

In conclusion we investigated shear localization in granular materials using a split-bottom Couette cell. While previous studies in similar geometries dealt with an open top boundary, here we confined the system by means of a top plate that applies an extra pressure on the granular pile, affecting the local stress and effective friction in the bulk of material. We studied the role of applied pressure on shear banding and demonstrated how shear zone characteristics, such as its center position and width, change upon varying the external load. We found that increasing external pressure promotes closed shear zones similarly to filling height. Moreover, previous studies mainly considered a simpler case of rough boundaries - by gluing a layer of grains to the container walls - to prevent slip. Here, we used smooth walls to allow for axial slip at the bottom plate and analyzed its influence on the emerged flow profiles. For a systematic study of the role of boundary roughness on granular rheology, we varied the ratio of the effective friction coefficients in the bulk and near the bottom plate in numerical simulations based on a variational approach to minimize the energy dissipation. We also numerically studied the role of grain size, as the material properties are known to influence the effective friction and response of granular materials in general and particularly the shear localization in Couette geometries.

Our results suggest that by tuning the boundary roughness and applied external pressure on a granular pile, the flow profile at a given filling height can be controlled to some extent. Understanding the rheology and mechanisms of shear localization in granular matter under confinement is crucial for industrial applications, e.g. in pharmaceutics and powder technology, as well as in geophysics. Shear localization can initiate avalanches, earthquakes, and faulting when the sheared layer withstands the weight of the upper layers. The results also once again highlight the applicability of the fluctuatingband variational approach with a self-organized random potential to describe granular shear flows. A possible application is the refraction of the shear zones when passing several layers with different confining pressures and material properties.

\section{ACKNOWLEDGMENTS}

We thank Matthias Schröter and Wolfgang Losert for fruitful discussions and comments. J.T. acknowledges support by Hungarian National Research, Development and Innovation Office (NKFIH), under Grant No. OTKA K 116036, by the BME IE-VIZTKP2020.
* shaebani@lusi.uni-sb.de

[1] A. Kudrolli, Rep. Prog. Phys. 67, 209 (2004).

[2] I. S. Aranson and L. S. Tsimring, Rev. Mod. Phys. 78, 641 (2006).

[3] R. Moosavi, M. Maleki, M. R. Shaebani, J. C. RuizSuárez, and E. Clément, EPL 107, 34006 (2014).

[4] A. Fall, A. Lemaître, F. m. c. Bertrand, D. Bonn, and G. Ovarlez, Phys. Rev. Lett. 105, 268303 (2010).

[5] M. Otsuki and H. Hayakawa, Phys. Rev. E 101, 032905 (2020).

[6] P. Schall and M. van Hecke, Annu. Rev. Fluid Mech. 42, 67 (2010)

[7] J. A. Dijksman and M. van Hecke, Soft Matter 6, 2901 (2010).

[8] F. Fan, E. J. R. Parteli, and T. Pöschel, Phys. Rev. Lett. 118, 218001 (2017).

[9] D. M. Mueth, G. F. Debregeas, G. S. Karczmar, P. J. Eng, S. R. Nagel, and H. M. Jaeger, Nature 406, 385 (2000).

[10] R. Moosavi, M. R. Shaebani, M. Maleki, J. Török, D. E. Wolf, and W. Losert, Phys. Rev. Lett. 111, 148301 (2013).

[11] D. Fenistein and M. van Hecke, Nature 425, 256 (2003).

[12] D. Fenistein, J. W. van de Meent, and M. van Hecke, Phys. Rev. Lett. 92, 094301 (2004).

[13] X. Cheng, J. B. Lechman, A. Fernandez-Barbero, G. S. Grest, H. M. Jaeger, G. S. Karczmar, M. E. Möbius, and S. R. Nagel, Phys. Rev. Lett. 96, 038001 (2006).

[14] P. Jop, Y. Forterre, and O. Pouliquen, Nature 441, 727 (2006).

[15] Y. Forterre and O. Pouliquen, Annu. Rev. Fluid Mech. 40, 1 (2008).

[16] D. Fenistein, J.-W. van de Meent, and M. van Hecke, Phys. Rev. Lett. 96, 118001 (2006).

[17] T. Unger, J. Török, J. Kertész, and D. E. Wolf, Phys. Rev. Lett. 92, 214301 (2004).

[18] J. Török, T. Unger, J. Kertész, and D. E. Wolf, Phys. Rev. E 75, 011305 (2007).

[19] D. L. Henann and K. Kamrin, Proc. Natl. Acad. Sci. USA 110, 6730 (2013).

[20] S. Li and D. L. Henann, Phys. Rev. E 102, 022908 (2020).

[21] M. Kobayakawa, S. Miyai, T. Tsuji, and T. Tanaka, Phys. Rev. E 98, 052907 (2018).

[22] M. R. Shaebani, M. Madadi, S. Luding, and D. E. Wolf, Phys. Rev. E 85, 011301 (2012).

[23] M. M. Bandi, P. Das, O. Gendelman, H. G. E. Hentschel, and I. Procaccia, Granular Matter 21, 40 (2019).

[24] P. Das, H. G. E. Hentschel, and I. Procaccia, Phys. Rev. E 99, 050902 (2019).

[25] M. R. Shaebani, J. Boberski, and D. E. Wolf, Granular Matter 14, 265 (2012).

[26] A. Singh, V. Magnanimo, K. Saitoh, and S. Luding, New J. Phys. 17, 043028 (2015).

[27] N. Murdoch, B. Rozitis, S. F. Green, T.-L. de Lophem, P. Michel, and W. Losert, Granular Matter 15, 129 (2013).

[28] M. R. Shaebani, T. Unger, and J. Kertész, Phys. Rev. E 76, 030301 (2007).

[29] C. Goldenberg and I. Goldhirsch, Nature 435, 188 (2005). 
[30] M. R. Shaebani, T. Unger, and J. Kertész, Phys. Rev. E 79, 052302 (2009).

[31] G. D. R. MiDi, Eur. Phys. J. E 14, 341 (2004).

[32] D. L. Blair, N. W. Mueggenburg, A. H. Marshall, H. M. Jaeger, and S. R. Nagel, Phys. Rev. E 63, 041304 (2001).

[33] S. Luding, Particul. Sci. Technol. 26, 33 (2007).

[34] T. Börzsönyi, B. Szabó, G. Törös, S. Wegner, J. Török, E. Somfai, T. Bien, and R. Stannarius, Phys. Rev. Lett. 108, 228302 (2012).

[35] G. Wortel, T. Börzsönyi, E. Somfai, S. Wegner, B. Szabó, R. Stannarius, and M. van Hecke, Soft Matter 11, 2570 (2015).

[36] D. Fischer, T. Börzsönyi, D. S. Nasato, T. Pöschel, and R. Stannarius, New J. Phys. 18, 113006 (2016).

[37] M. R. Shaebani, T. Unger, and J. Kertész, Int. J. Mod. Phys. C 20, 847 (2009).

[38] G. Chambon, J. Schmittbuhl, A. Corfdir, J. P. Vilotte, and S. Roux, Phys. Rev. E 68, 011304 (2003).

[39] R. R. Hartley and R. P. Behringer, Nature 421, 928 (2003).
[40] L. Onsager, Phys. Rev. 37, 405 (1931).

[41] L. Onsager, Phys. Rev. 38, 2265 (1931).

[42] R. Baker and M. Garber, Géotechnique 28, 395 (1978).

[43] I. Einav and M. Randolph, Géotechnique 56, 501 (2006).

[44] T. Unger, Phys. Rev. Lett. 98, 018301 (2007).

[45] B. Szabó, J. Török, E. Somfai, S. Wegner, R. Stannarius, A. Böse, G. Rose, F. Angenstein, and T. Börzsönyi, Phys. Rev. E 90, 032205 (2014).

[46] T. Börzsönyi, T. Unger, and B. Szabó, Phys. Rev. E 80, 060302 (2009).

[47] Z. Shojaaee, M. R. Shaebani, L. Brendel, J. Török, and D. E. Wolf, J. Comput. Phys. 231, 612 (2012).

[48] S. Plimpton, J. Comput. Phys. 117, 1 (1995), URL https://lammps.sandia.gov/

[49] M. R. Shaebani, T. Unger, and J. Kertész, Phys. Rev. E 78, 011308 (2008). 